
28 June - 03 July 2010, Montpellier

\title{
Studies of open charm and charmonium production at LHCb
}

Yu. Guz (IHEP Protvino), on behalf of the LHCb collaboration

02.07.2010 


\section{The LHCb detector and run 2010/11}

(see also talks of F. Dettori and J. Dickens) 


\section{The LHCb detector}

Search for New Physics in CP violation and Rare Decays

Optimized for B-physics.

- The bb production cross section is large, $\sigma(b \bar{b}) \sim 300-500 \mu b$ at $\sqrt{ } s=7-14 \mathrm{TeV}$

b

- The bb production is sharply peaked forward-backward.

$\mathrm{LHCb}$ is a single arm detector $1.9<|n|<4.9$ designed to work at $\mathcal{L} \approx 2 \cdot 10^{32} \mathrm{~cm}^{-2} \mathrm{~s}^{-1} \sqrt{\mathrm{s}}=14 \mathrm{TeV}$

"Nominal LHCb year": $2 \mathrm{fb}^{-1}$

\section{Muon System}

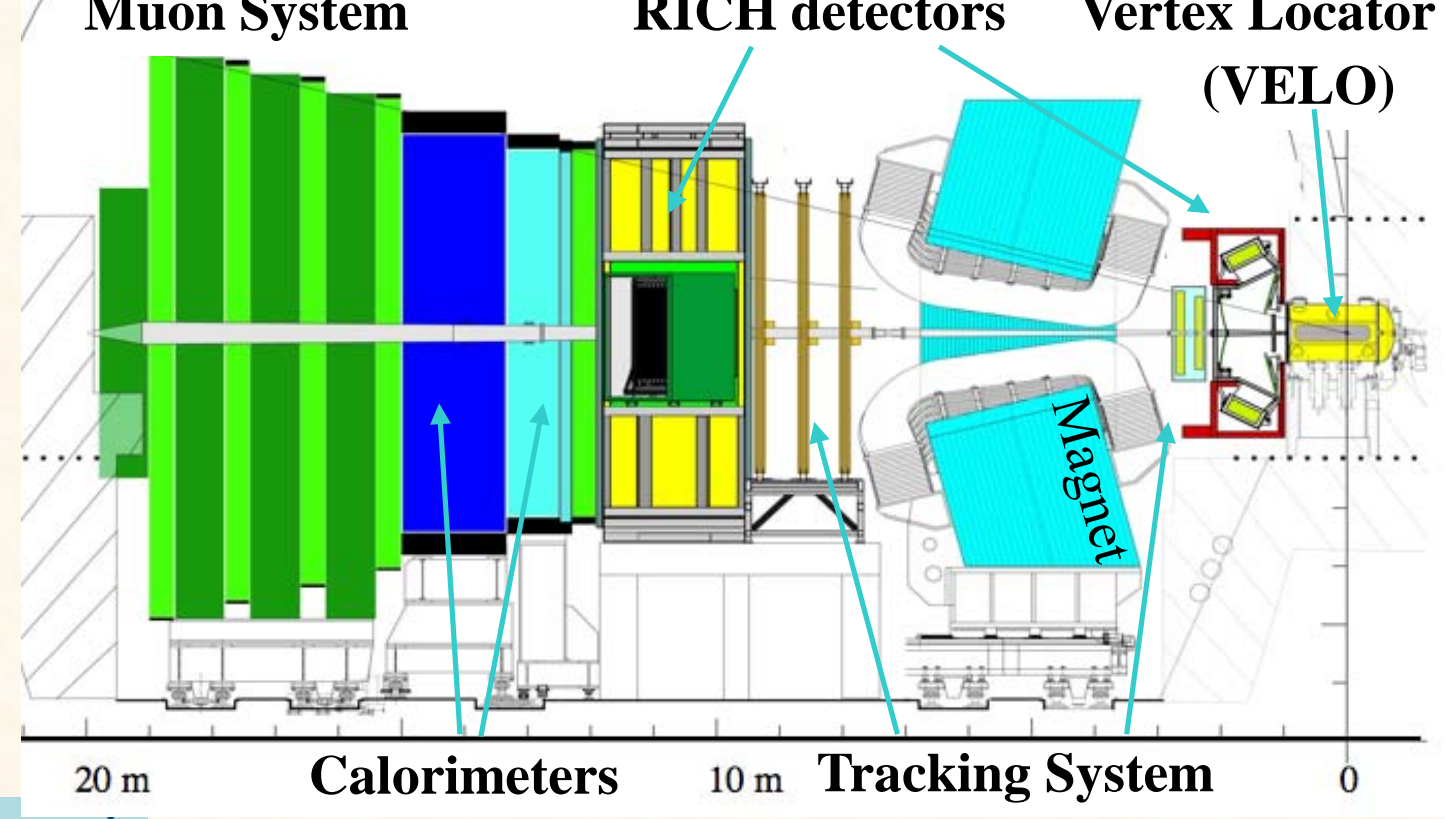

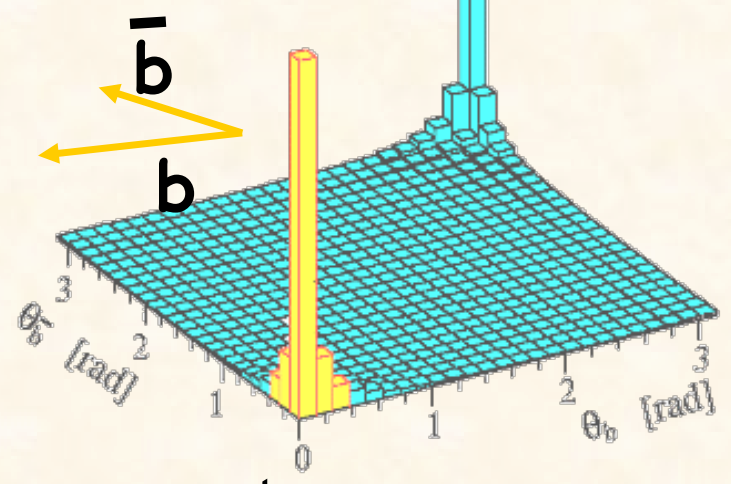

Main components:

- silicon strip vertex detector surrounding the IP

- magnet

- tracker stations (inner area: silicon; outer: straw tubes)

- two RICH detectors

- EM calorimeter with preshower

- muon system 
Hardware Level-0 trigger followed by two-stage software High Level Trigger, HLT1 and HLT2

- L0 requires presence of a high $p_{T} \operatorname{object~}\left(h, \mu, \mu \mu, Y, e^{ \pm}\right)$ in CALO and Muon system

- HLT1 confirms L0 objects: associates them with reconstructed tracks, especially with those displaced from the PV

- HLT2 uses reconstructed objects for exclusive selections with clear signature

A suite of selections for key event types for charm physics is present in HLT2.

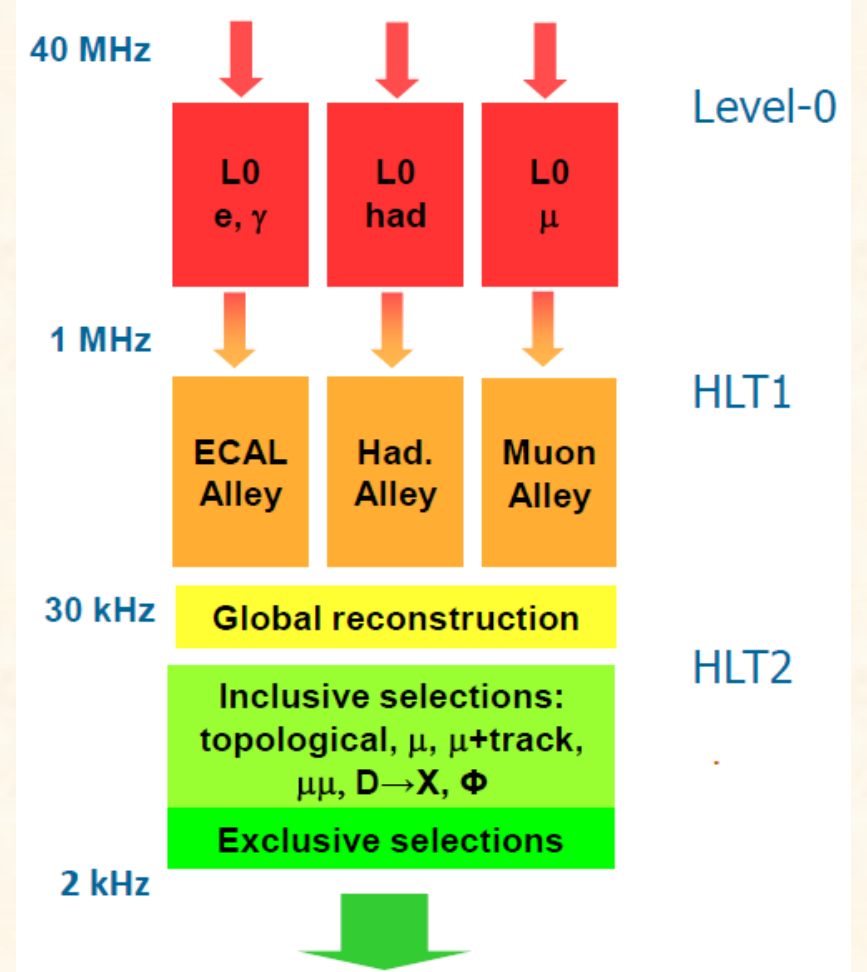

At the design luminosity $\left(2 \cdot 10^{32} \mathrm{~cm}^{-2} \mathrm{~s}^{-1}\right)$ the thresholds will be optimized for B-physics, the efficiency for decays of prompt D's being 10\%

Depending on luminosity, the LO and HLT1 thresholds can be tuned such that not to exceed maximal throughput of the systems. 


\section{LHCb run 2010-2011}

LHC is running smoothly since its startup, gradually increasing its luminosity. It is running at $\mathrm{E}_{\mathrm{CM}}=7 \mathrm{TeV}$ (the $c \bar{C}$ production cross section expected to be $\sim 0.57$ of that at $14 \mathrm{TeV}$ )

- by now, $\mathcal{L} \approx 7 \cdot 10^{29} \mathrm{~cm}^{-2} \mathrm{~s}^{-1}$ achieved

- by the end of 2010 it is supposed to achieve:

- $\mathcal{L} \approx 10^{31} \mathrm{~cm}^{-2} \mathrm{~s}^{-1}, \int_{2010} \mathcal{L} \mathrm{dt} \approx 100 \mathrm{pb}^{-1}$

- $-10^{11} \quad c \bar{c}$ events produced

- in 2011:

- reach LHCb design luminosity, $\mathcal{L} \approx 2 \cdot 10^{32} \mathrm{~cm}^{-2} \mathrm{~s}^{-1}$

- $\int_{2011} \mathcal{L} \mathrm{dt} \approx 1 \mathrm{fb}^{-1}$

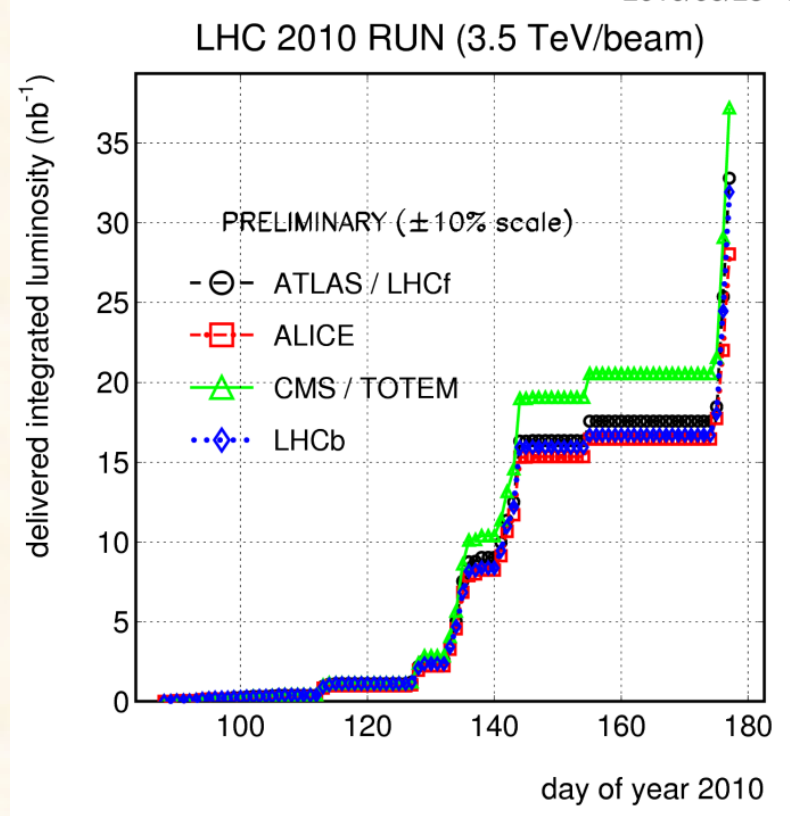

In 2010, with luminosities below few $10^{31} \mathrm{~cm}^{-2} \mathrm{~s}^{-1}$, LHCb works with much lower trigger thresholds and factor 4-5 higher efficiencies for prompt D's

$\rightarrow$ great opportunities for charm physics! 


\section{LHCb run 2010-2011}

At present, charmonium ant open charm production signals are clearly seen in LHCb.

In 2010/11, LHCb expects to produce results in several topics of charm physics. The work is ongoing on:

- differential cross section of (prompt) charmonium and open charm states production at $\sqrt{ } \mathrm{s}=7 \mathrm{TeV}$

- physics of charm:

1. $D^{0}-\bar{D}^{0}$ oscillation parameters from tagged $D^{0} \rightarrow K \pi$

2. $y_{C P}$ from lifetime differences in $\mathrm{D}^{0} \rightarrow \mathrm{K} \pi, \mathrm{KK}, \pi \pi$

3. direct CPV from Dalitz plot analysis of SCS decays $D^{ \pm} \rightarrow K^{+} K^{-} \pi^{ \pm}\left(\pi^{+} \pi^{-} \pi^{ \pm}\right)$

4. T violation from $D^{0} \rightarrow K^{+} K^{-} \pi^{+} \pi^{-}$decay (not discussed here)

5. rare decays: improve upper limit on $B R\left(D^{0} \rightarrow \mu \mu\right)$ (not discussed here)

6. ... 


\section{Charmonium}
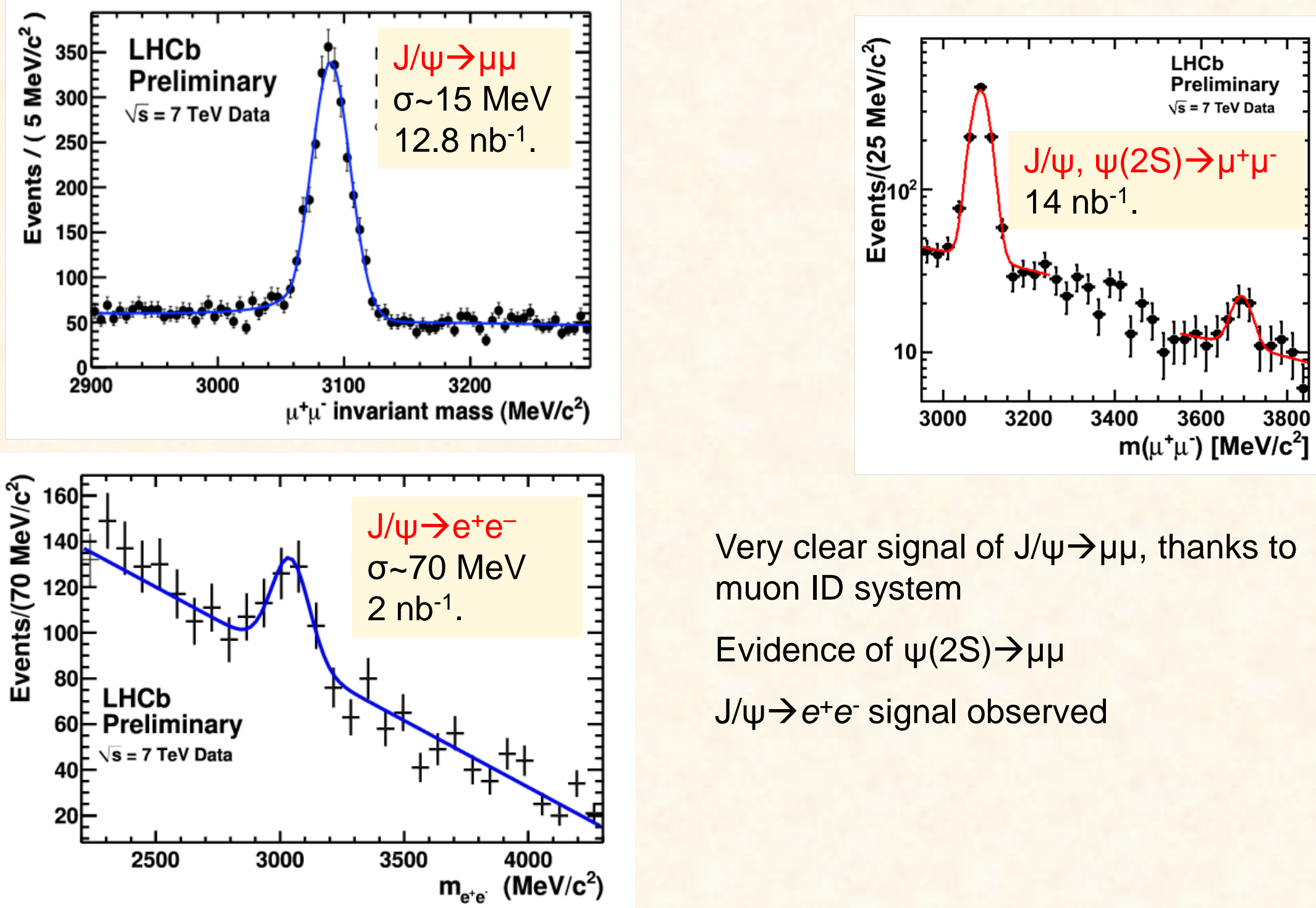

Very clear signal of $\mathrm{J} / \psi \rightarrow \mu \mu$, thanks to muon ID system

Evidence of $\psi(2 S) \rightarrow \mu \mu$

$\mathrm{J} / \Psi \rightarrow e^{+} e^{-}$signal observed 


\section{Untagged $\mathrm{D} 0 \rightarrow \mathrm{KK}, \mathrm{K} \pi, \pi \pi$}
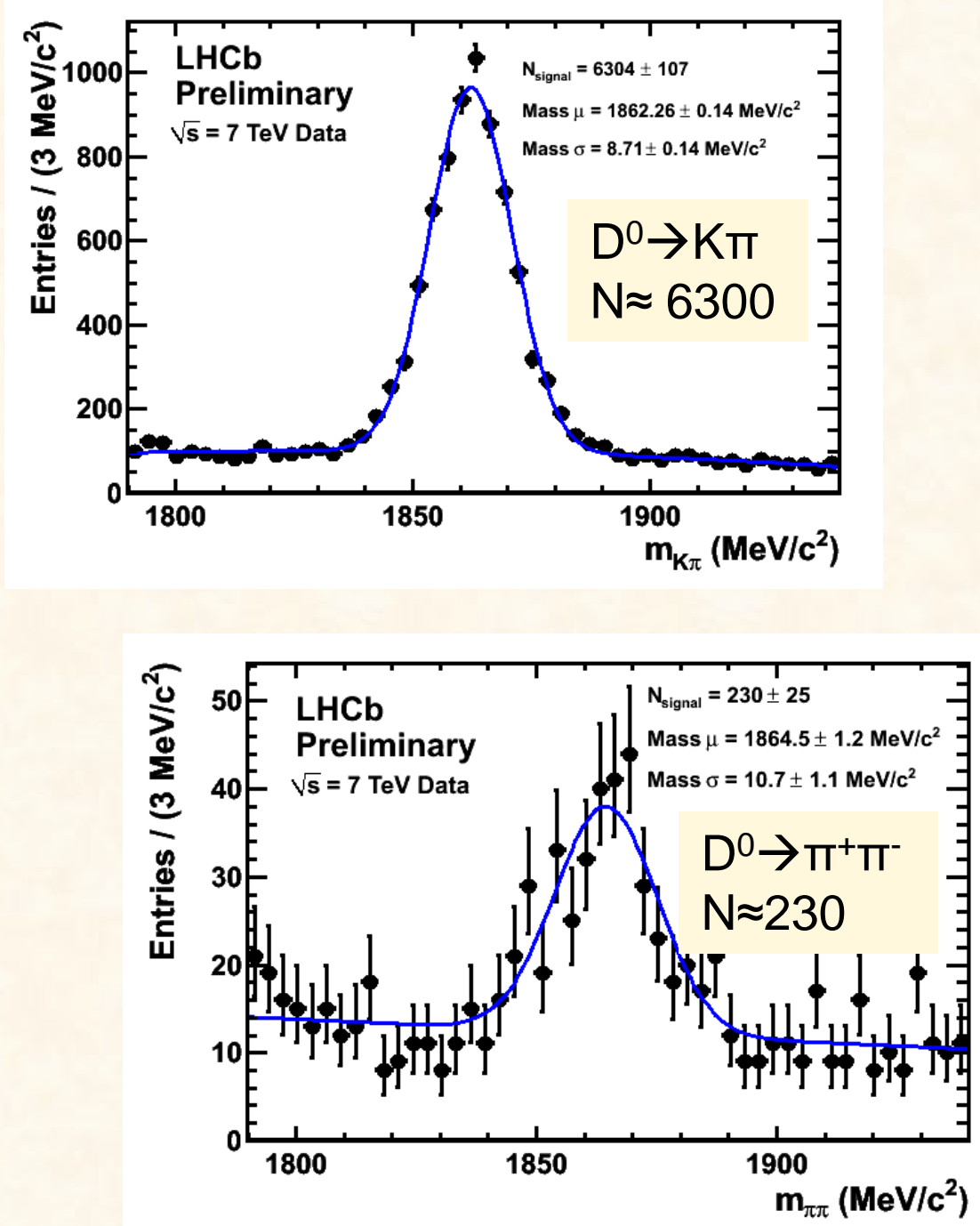

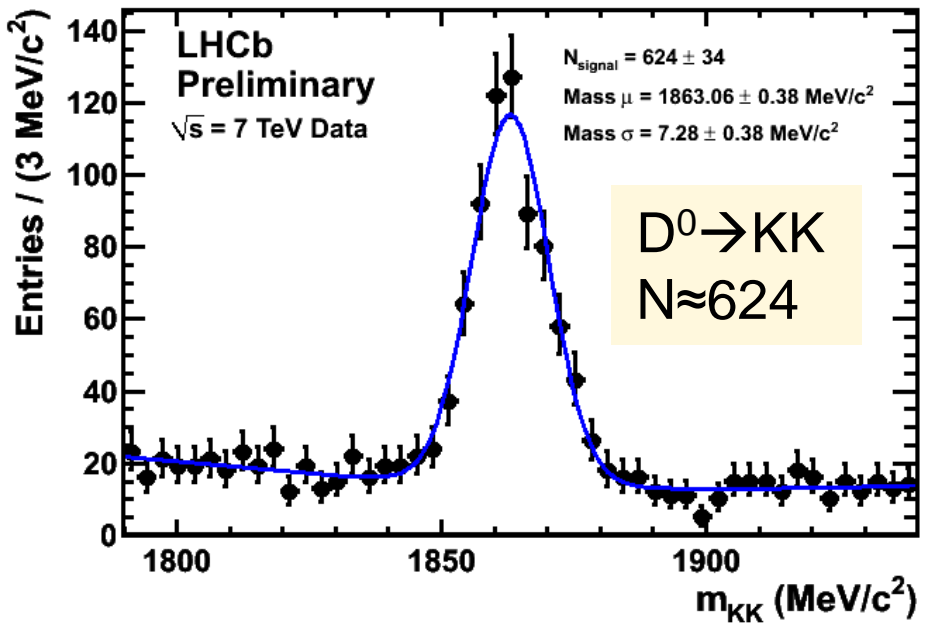

Data of $\sim 2.7 \mathrm{nb}^{-1}$.

Very clear $\mathrm{D} 0 \rightarrow \mathrm{K} \pi \mathrm{D} 0 \rightarrow \mathrm{KK}$ signals, benefitting from RICH PID

Low lumi data, trigger efficiency $\varepsilon \sim 100 \%$

Expected in $2010(\varepsilon \sim 40 \%)$ :

$\mathrm{N}\left(\mathrm{D}^{0}\right.$ untagged $) \approx 9.3 \cdot 10^{7}$ events 


\section{Tagged, $D^{*} \rightarrow \pi_{\text {slow }} D(K \pi)$}
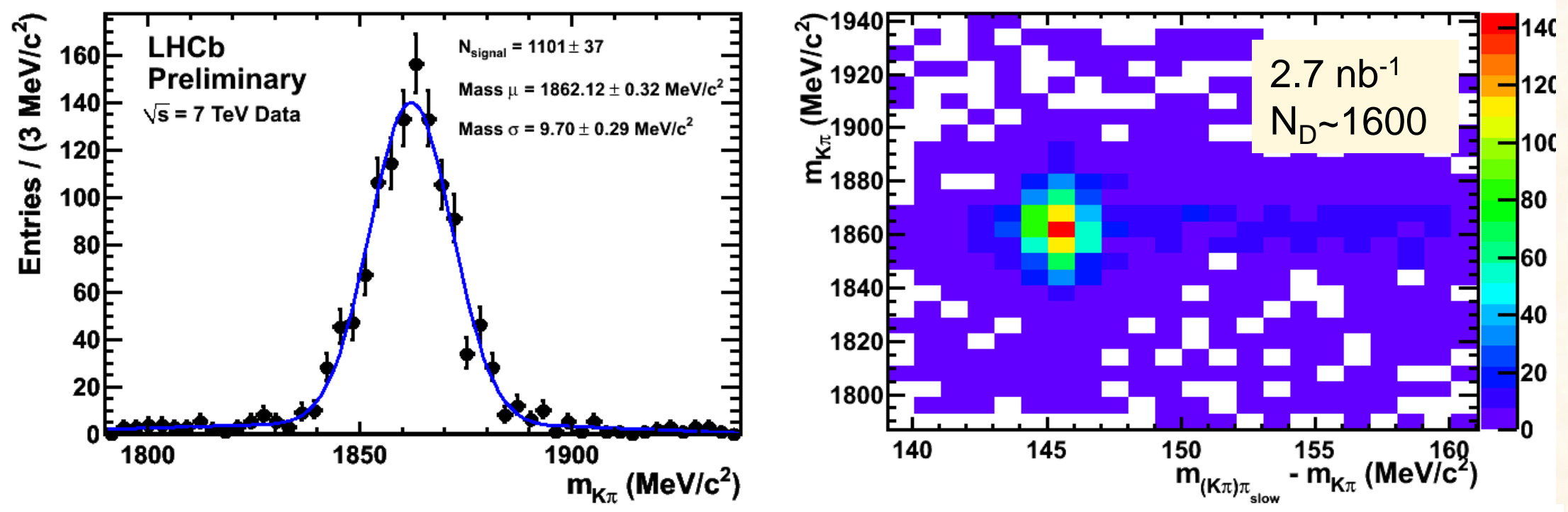

(in projections: $\pm 2 \sigma$ cut on non plotted variable)

Expected in $2010\left(100 \mathrm{pb}^{-1}\right)$ :

$\mathrm{N}\left(\mathrm{D}^{0}\right.$ tagged $) \approx 1.7 \cdot 10^{7}$ events

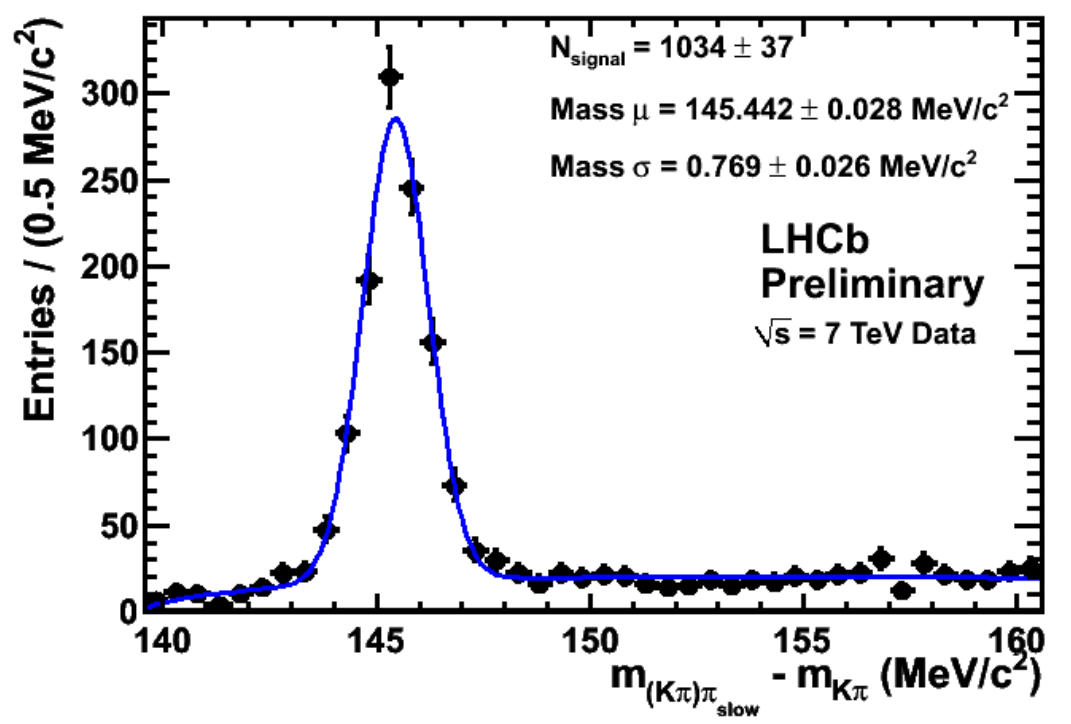




\section{Other open charm signals}
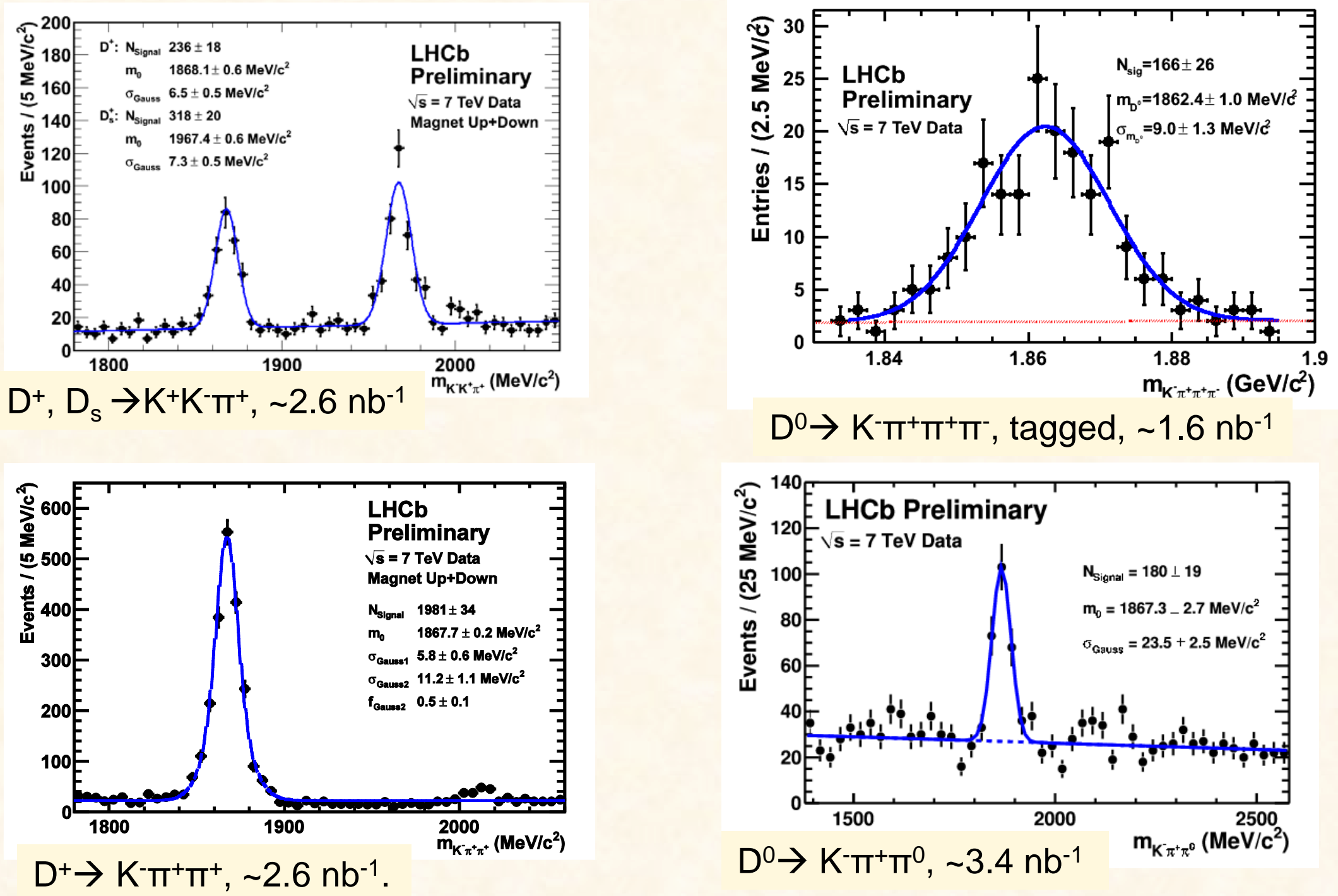


\section{Charmonium and open charm production studies}




\section{$J / \Psi$ production studies}

- $\mathrm{J} / \Psi$ production mechanism in hadronic collisions is not well understood. Measurement at LHC energies will give another piece of information.

- LHCb MC tuning: important for later analysis

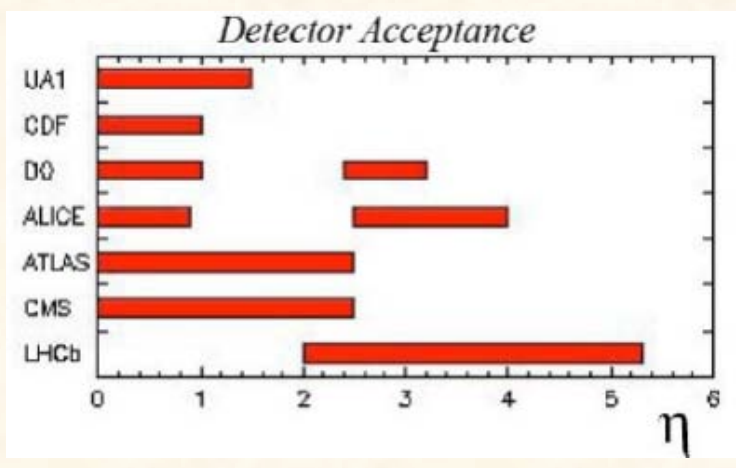

- $\mathrm{J} / \Psi$ 's are abundantly produced at LHC energies ( $\sim 0.1 \mathrm{mb})$

- LHCb probes the kinematical area not accessible by other LHC experiments, where the theoretical predictions are less accurate

3 main sources of $\mathrm{J} / \Psi$ production:

$>$ direct production in pp collision

$>$ from decays of heavier charmonium states<smiles>CCC(CC)OCCO</smiles>

* from b-hadron decays
Can be distinguished by measuring the decay vertex displacement from PV

The goal is to measure the (prompt) $\mathrm{J} / \Psi$ production cross section and polarization as a function of transverse momentum $\left(\mathrm{p}_{\mathrm{T}}\right)$ and rapidity $(\mathrm{y})$, using $\mathrm{J} / \psi \rightarrow \mu^{+} \mu^{-}$decay mode.

Namely, we aim at $\mathrm{O}(10 \%)$ accuracy in $5 \times 12$ bins in $2<y<4.5, \quad 0<p_{T}<12 \mathrm{GeV} / \mathrm{c}$, first results at $\sim 10-20 \mathrm{nb}^{-1}$. 


\section{$\mathrm{J} / \Psi$ distributions}
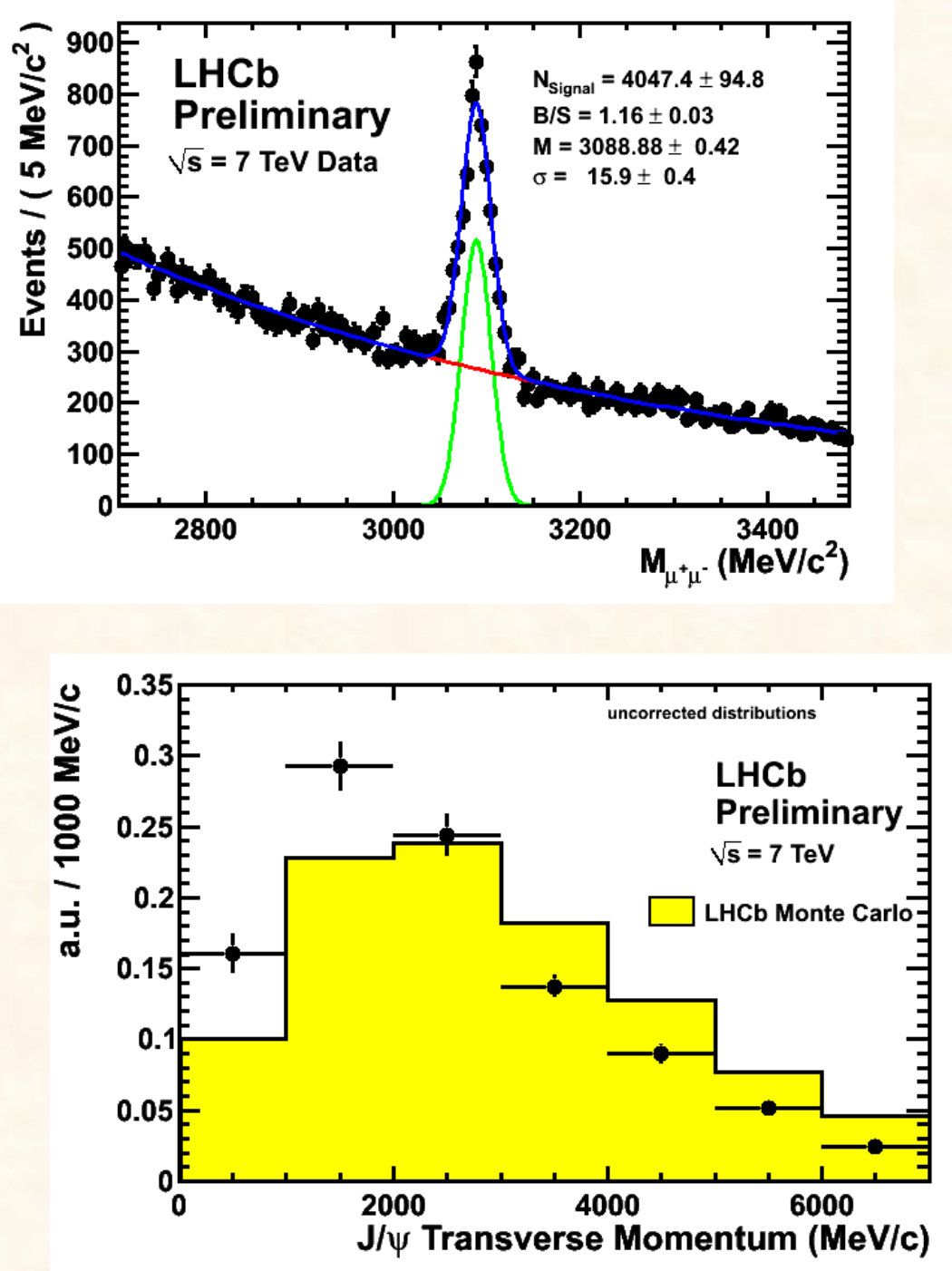

Loose cuts suitable for the cross section determination

$\sim 4 \cdot 10^{3}$ reconstructed $\mathrm{J} / \psi^{\prime}$ 's in $14 \mathrm{nb}^{-1}$ :

$\sim 2 \cdot 10^{7}$ reconstructed $\mathrm{J} / \Psi$ 's expected in 2010 (in $100 \mathrm{pb}^{-1}$ )

The 1D J/ $\Psi$ production spectra obtained from bin-by-bin fitting of $\mathrm{M}_{\mu \mu}$ distribution. Good agreement with MC in y; some difference seen in $\mathrm{p}_{\mathrm{T}}$.

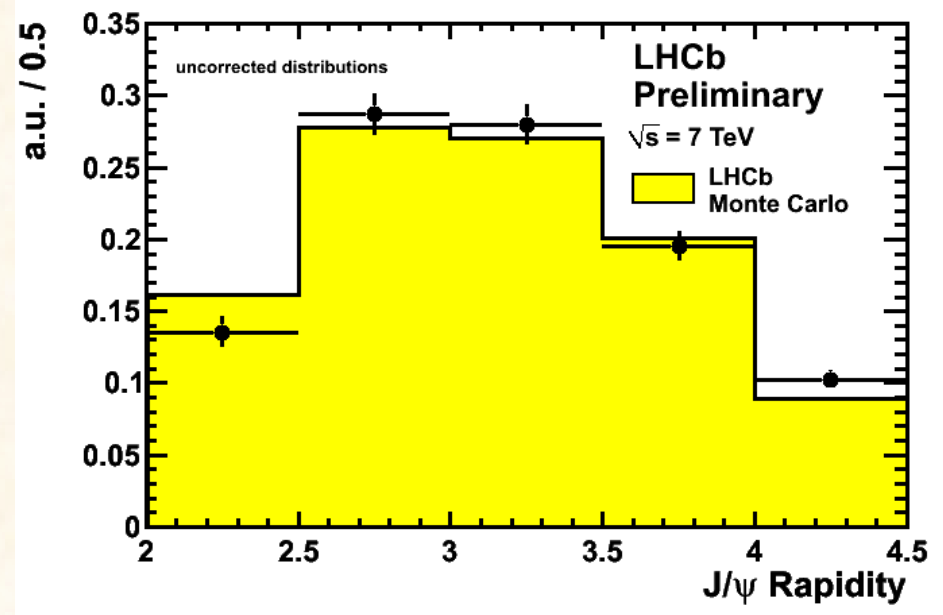




\section{$J / \Psi:$ prompt and secondary production}
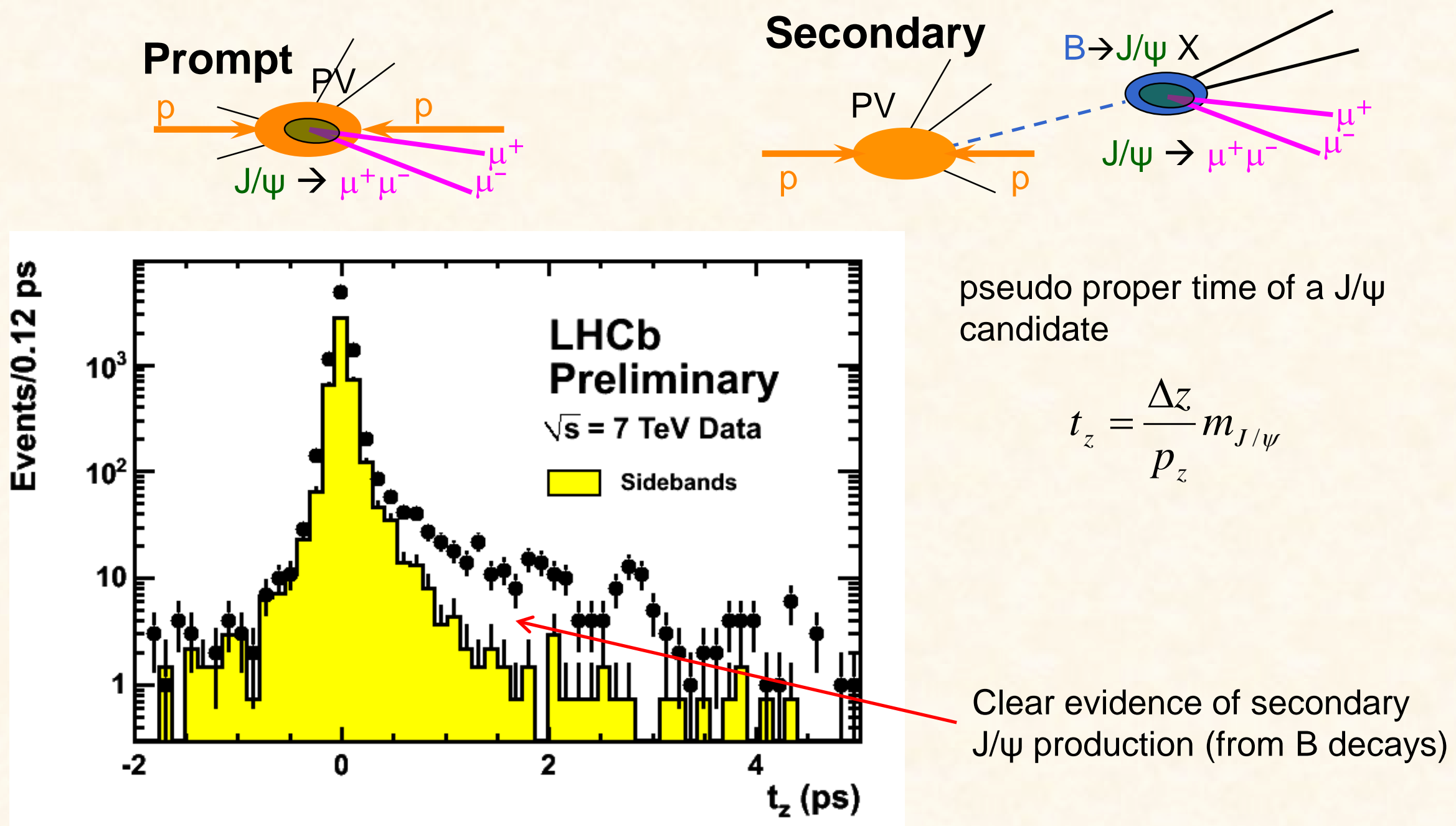


\section{$J / \Psi:$ further steps}

Full analysis will be possible with $10-20 \mathrm{pb}^{-1}$ (several $10^{6}$ events).

For the cross section measurement, one needs precise knowledge of the detector efficiency in the region of interest, which includes efficiency of:

- trigger

- track reconstruction

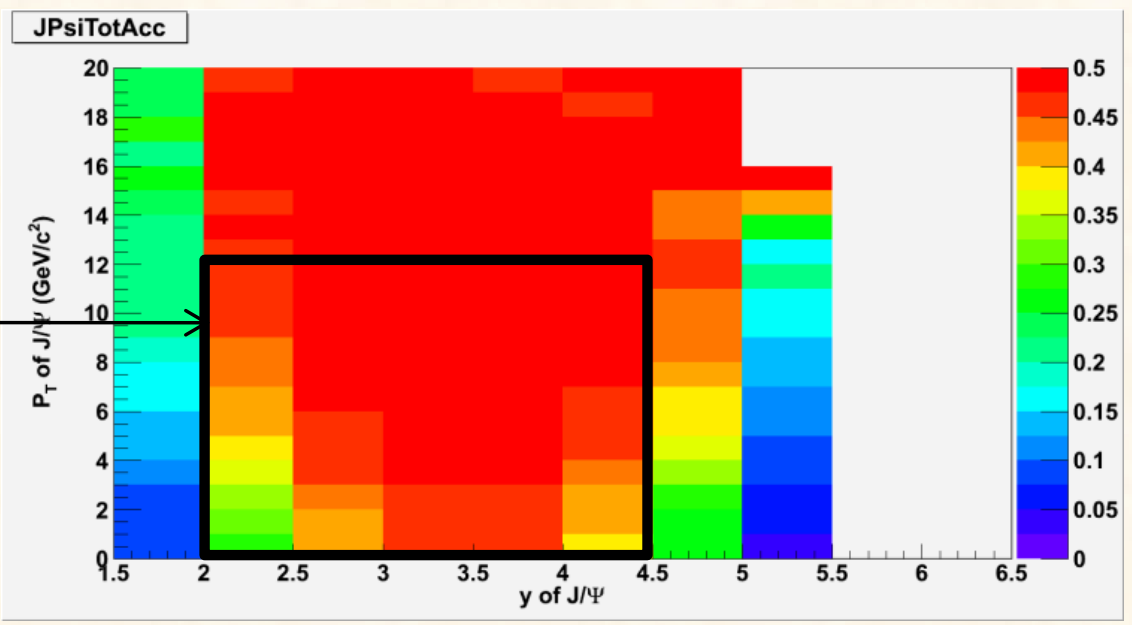

- $\mu$ ID

which are to be tuned on real data.

NB the polarization measurement is especially sensitive to the detector knowledge: up to $20 \%$ polarization dependence in some $\left(p_{T}, y\right)$ bins.

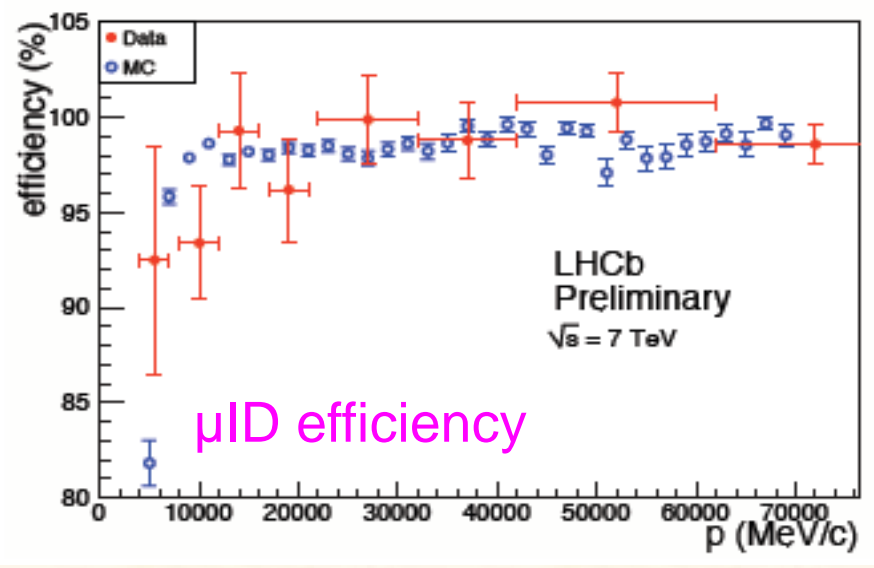

$\mu$ ID efficiency determined with "tag and probe" method using $\mathrm{J} / \psi \rightarrow \mu^{+} \mu^{-}$. 


\section{Open charm production studies}

Analyses are ongoing on measuring the production cross section of $\mathrm{D}^{0}, \mathrm{D}^{+}, \mathrm{D}_{\mathrm{S}}$ $(+c c)$ with the following fully-reconstructed hadronic decay modes:

$>\mathrm{D}^{0} \rightarrow \mathrm{K}^{-} \mathrm{\Pi}^{+}$;

$>\mathrm{D}^{+} \rightarrow \mathrm{K}^{-} \mathrm{T}^{+} \mathrm{T}^{+}$

$>\mathrm{D}^{*+} \rightarrow \pi^{+} \mathrm{D}^{0}\left(\mathrm{D}^{0} \rightarrow \mathrm{K}^{-} \Pi^{+}\right)$;

$>\mathrm{D}_{\mathrm{S}} \rightarrow \mathrm{K}^{-} \mathrm{K}^{+} \Pi^{+}$.

Situation similar to that in the charmonium production.

clear signals are observed in the early data

on the way to production measurements

separation of prompt and secondary contributions is trickier because of charm lifetime: possible on the basis of D meson IP 


\section{D mesons: prompt-secondary discrimination}
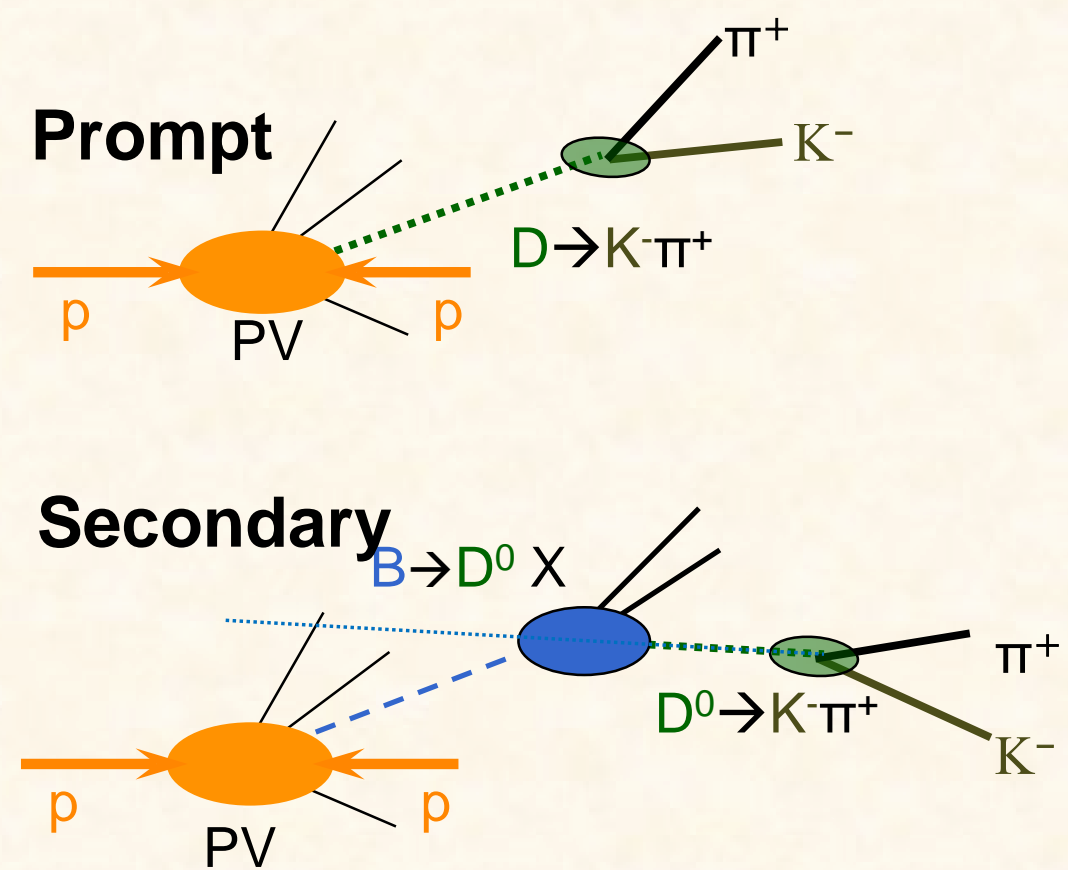

The D momentum

> Prompt: points to PV

> Secondary: does not point to PV in general

Separation of prompt and secondary contributions is possible on the basis of $D$

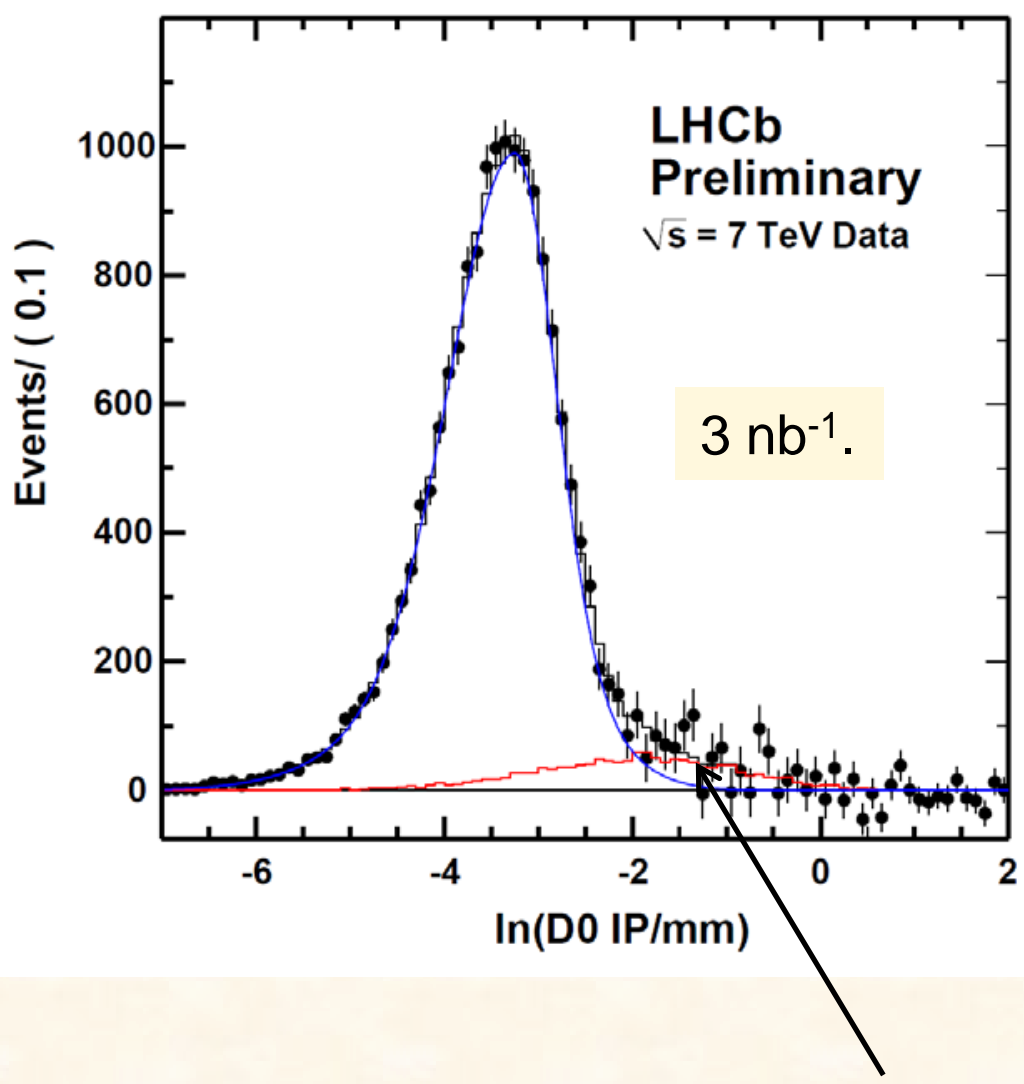
meson IP

$\sim 1300 D^{0}$ mesons produced in $\mathrm{B}$ decays 


\section{Prospects for Charm physics}




\section{$D^{0}-\bar{D}^{0}$ mixing}

Oscillation parameters : $\mathrm{x}=\left(\mathrm{M}_{1}-\mathrm{M}_{2}\right) / \Gamma, \mathrm{y}=\left(\Gamma_{1}-\Gamma_{2}\right) / 2 \Gamma, \Gamma=\left(\Gamma_{1}+\Gamma_{2}\right) / 2$

$D_{1}$ and $D_{2}$ are physical eigenstates, $\left|D_{1,2}\right\rangle=p\left|D^{0}\right\rangle \mp q\left|\overline{D^{0}}\right\rangle$

In SM predicted to be small, $(x, y) \sim 10^{-2}$, caused mainly

by long-distance effects

Measure the ratio of wrong sign $\mathrm{D}^{0} \rightarrow \mathrm{K}^{+} \Pi^{-}$decays (DCS) to right sign $\mathrm{D}^{0} \rightarrow \mathrm{K}^{-} \pi^{+}(\mathrm{CF})$ as a function of proper time: assuming $\mathrm{CP}$ conservation and $\left(\mathrm{x}^{2}+\mathrm{y}^{2}\right)<<1$ $R(t / \tau)=R_{D}+\sqrt{R_{D}} y^{\prime}(t / \tau)+\left(x^{\prime 2}+y^{\prime 2}\right) / 4 \cdot(t / \tau)^{2}$ (strong phase $\delta_{K_{\pi}}$ between DCS and CF: $(x, y) \rightarrow\left(x^{\prime}, y^{\prime}\right)$ ) (BaBaR 2007, CDF 2008)

World average: no-mixing excluded at $\sim 10.2 \sigma$ level (although no single observation $>5 \sigma$ )

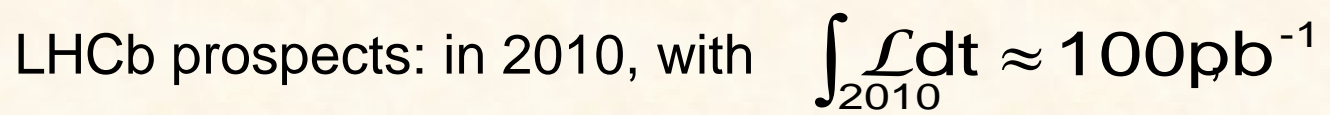
$\mathrm{N}_{\mathrm{RS}} \approx 1.7 \cdot 10^{7}, \mathrm{~N}_{\mathrm{ws}} \approx 6 \cdot 10^{4} 6 \rightarrow 5$ times more than BABAR+CDF Improvement expected in ( $\left.\mathrm{x}^{\prime}, \mathrm{y}^{\prime}\right)$ measurement precision
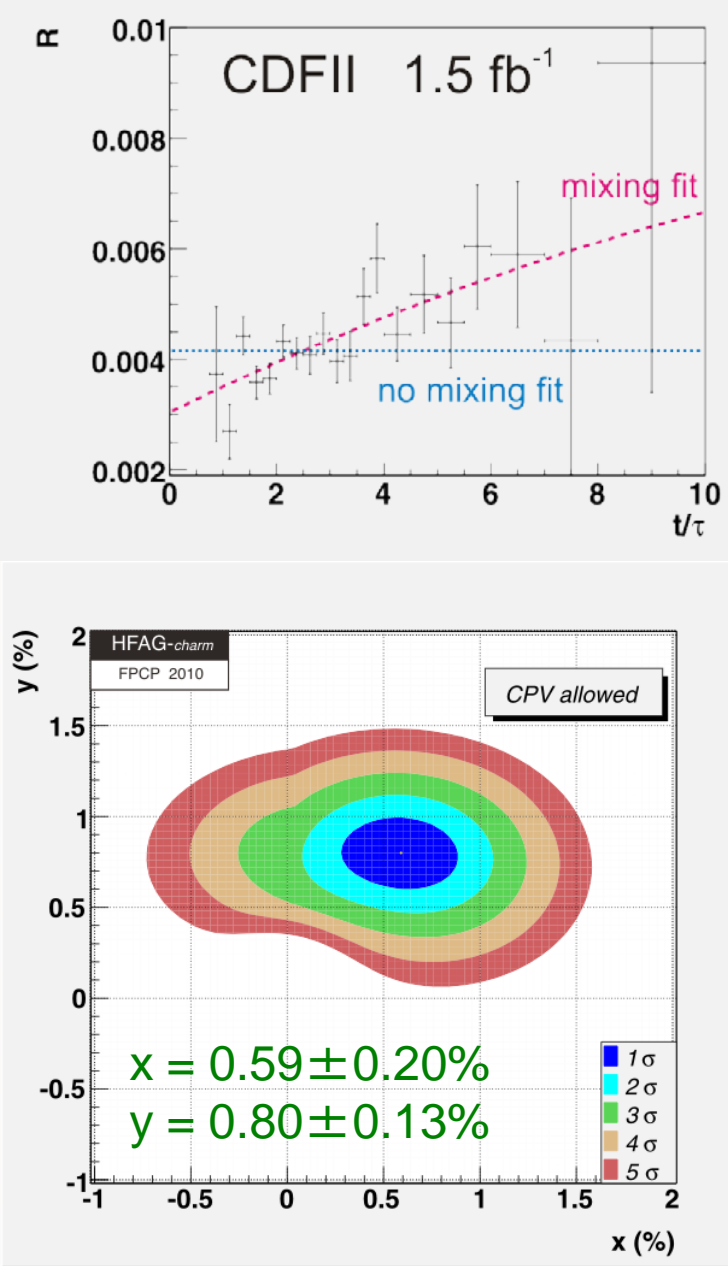


\section{CPV in mixing parameters}

SM can generate only tiny CPV effects in charm $<\mathrm{O}\left(10^{-3}\right)$ : any higher asymmetry is a sign of New Physics

Experimentally measurable e.g. through lifetime differences of $D^{0}\left(\overline{\mathrm{D}}^{0}\right)$ decays into CP-even $\left(h^{+} h^{-}, h=\{K, \pi\}\right)$ and CP-mixed CF $K^{-} \pi^{+}\left(K^{+} \pi^{-}\right)$final states:

$y_{C P}=\frac{\tau\left(D^{0} \rightarrow K^{-} \pi^{+}\right)}{\tau\left(D^{0} \rightarrow h h\right)}-1 \quad A_{\Gamma}=\frac{\tau\left(D^{0} \rightarrow h h\right)-\tau\left(\bar{D}^{0} \rightarrow h h\right)}{\tau\left(D^{0} \rightarrow h h\right)+\tau\left(\bar{D}^{0} \rightarrow h h\right)}$

(with CP conservation, $y_{C P}=y, A_{\Gamma}=0$ )

BaBaR 2009: $y_{C P}=[1.16 \pm 0.22$ (stat) \pm 0.18 (syst) $] \%$

Expected \# of (tagged) $\mathrm{D}^{0}$ decays in LHCb in 2010, with $100 \mathrm{pb}^{-1}$ :

$\mathrm{N}\left(\mathrm{D}^{0} \rightarrow \mathrm{K} \pi\right) \approx 1.7 \cdot 10^{7}$

$\mathrm{N}\left(\mathrm{D}^{0} \rightarrow \mathrm{KK}\right) \approx 1.3 \cdot 10^{6}$

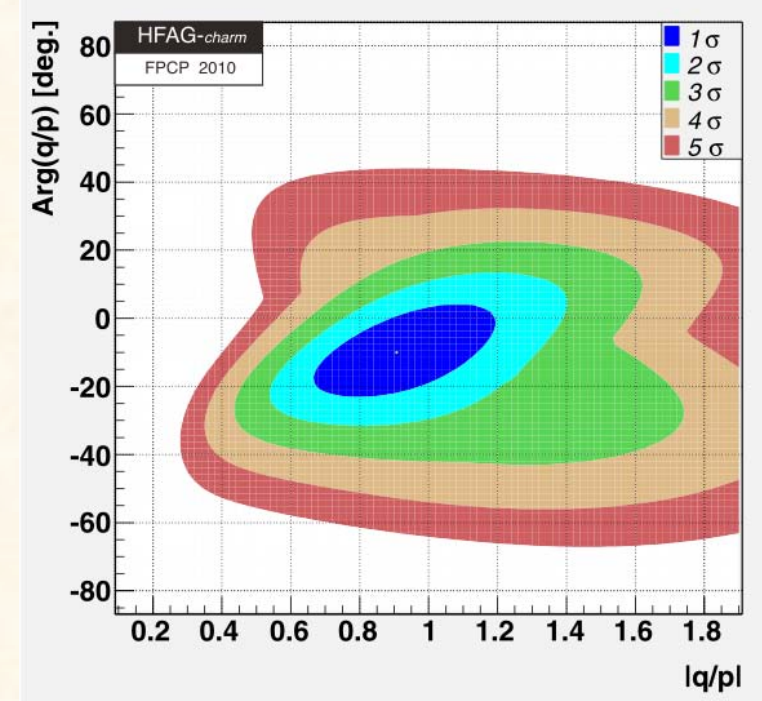

$\rightarrow 10$ times more than in the BABAR analysis 


\section{Direct CPV in SCS D decays}

New Physics can generate CP violation effects in SCS decays through loop contribution in the $c \rightarrow u$ transition.

3-body decays $\left(D^{ \pm} \rightarrow K^{+} K^{-} \pi^{ \pm}, \pi^{+} \pi^{-} \pi^{ \pm}\right)$are suitable for CPV searches, because not only $\mathrm{D}^{+} / \mathrm{D}^{-}$decay rate difference can be measured, but also local asymmetries in the Dalitz plot. (PRD80:096006,2009) .

Expected few $10^{6} D^{+} \rightarrow K^{+} K^{-} \pi^{+}(+c c)$ decays in 2010
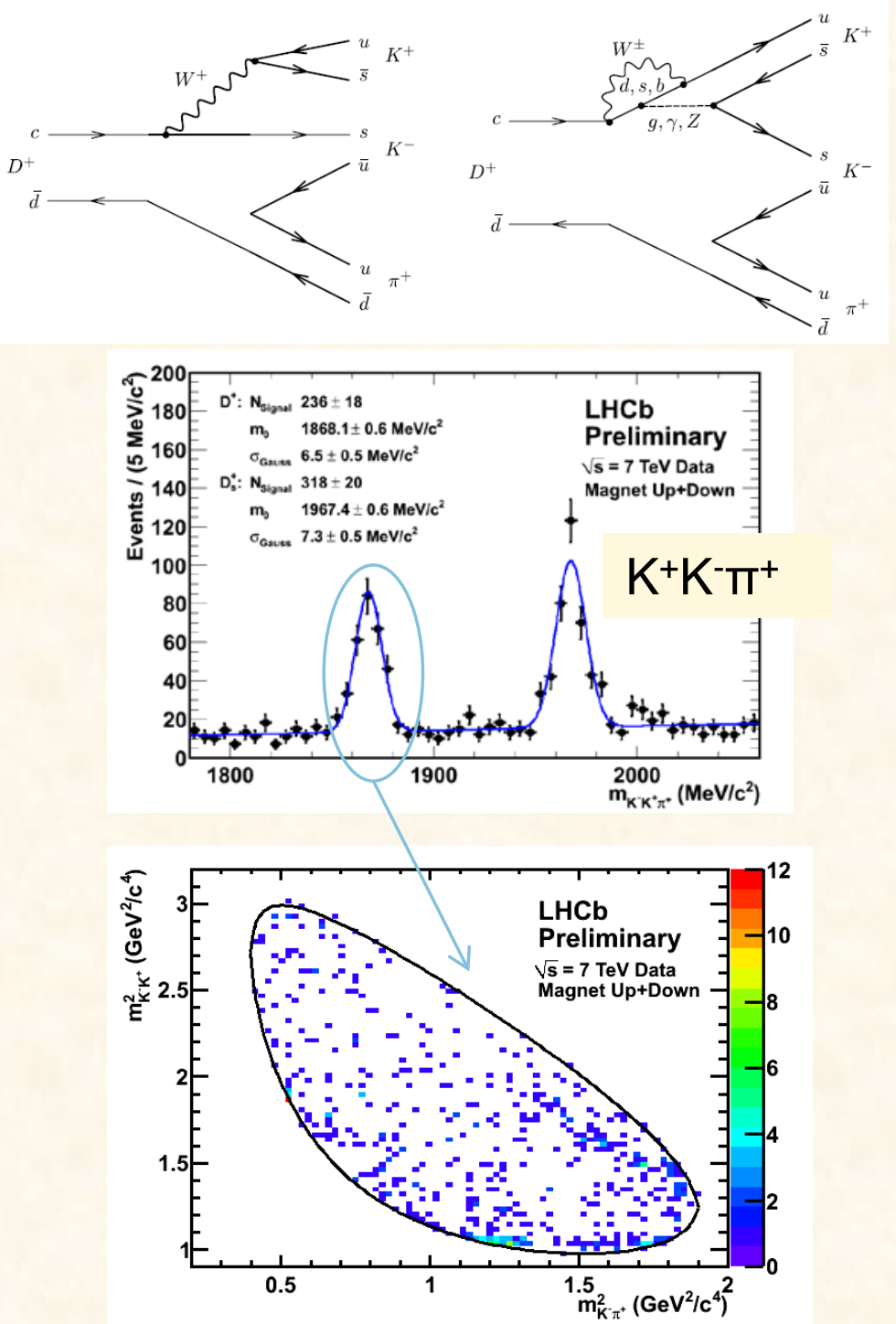


\section{Conclusions}

- LHCb is running successfully, many charmonium and open charm signals are observed now at $\sim 16 \mathrm{nb}-1$

- by the end 2010, LHCb will produce results on differential cross section of charmonium and open charm production at $3.5 \mathrm{TeV}$

- in 2010/2011 LHCb will be able to contribute into charm physics, in particular in what concerns measurements of $\mathrm{D}^{0}-\overline{\mathrm{D}}^{0}$ mixing and $\mathrm{CP}$ violation parameters in the charm sector. 


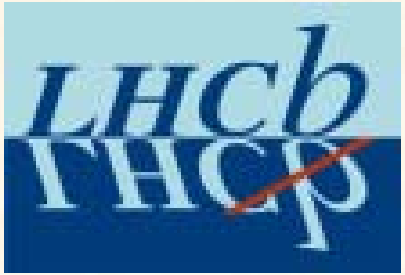

\section{Backup}




\section{Luminosity measurement in $\mathbf{2 0 1 0}$}

In 2010, luminosity will be estimated from beam properties

$$
L=f \sum_{i=1}^{N} \frac{n_{1 i} n_{2 i}}{4 \pi \sigma_{X i} \sigma_{Y i}}
$$

$\mathrm{N}$ - number of bunches

$f$ - collision frequency

$\mathrm{n}_{1 \mathrm{i}}, \mathrm{n}_{2 \mathrm{i}}$-- \# of protons in bunches

$\sigma_{\mathrm{Xi}}, \sigma_{\mathrm{Yi}_{\mathrm{i}}}-$ transverse bunch sizes

LHCb preliminary $2009, \mathrm{E}_{\mathrm{CM}}=0.9 \mathrm{TeV}$
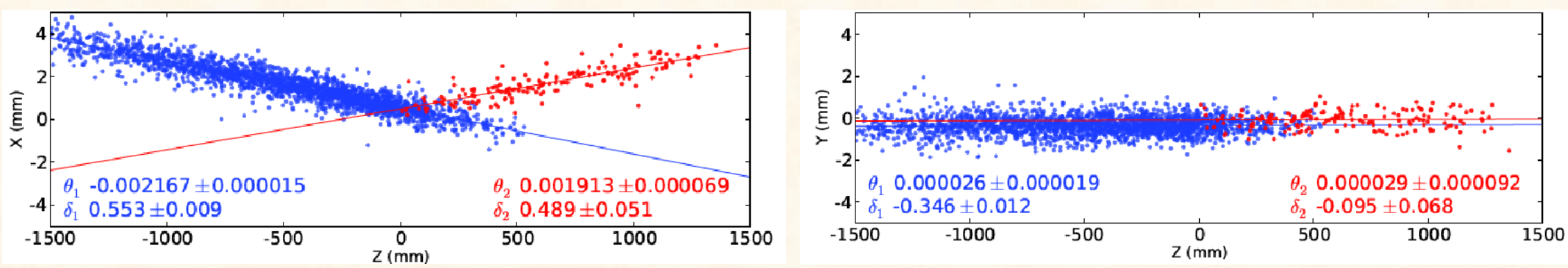

Determined with $\sim 15 \%$ accuracy in 2009 (dominated by the bunch current measurement uncertainty). In $20105-10 \%$ precision is expected. 


\section{Tracking system performance}

\section{- Tracking system:}

$>$ IP resolution: $\sim 16 \mu \mathrm{m}$ in $X$ and $Y(\sim 12 \mu \mathrm{m}$ in $\mathrm{MC})$

$>\mathrm{PV}: \sim 15 \mu \mathrm{m}$ for $X$ and $Y, \sim 90 \mu \mathrm{m}$ for $Z$

$>$ Mass resolutions of reconstructed particles - as expected from MC:

- $\mathrm{K}_{\mathrm{S}} \rightarrow \pi^{+} \pi^{-}: \mathrm{m}=497.2 \mathrm{MeV}, \sigma=6.6 \mathrm{MeV}$

- $\wedge \rightarrow$ пр : $m=1115.7 \mathrm{MeV}, \sigma=2.9 \mathrm{MeV}$

- $\equiv \rightarrow \wedge \pi: m=1321.9 \mathrm{MeV}, \sigma=2.7 \mathrm{MeV}$

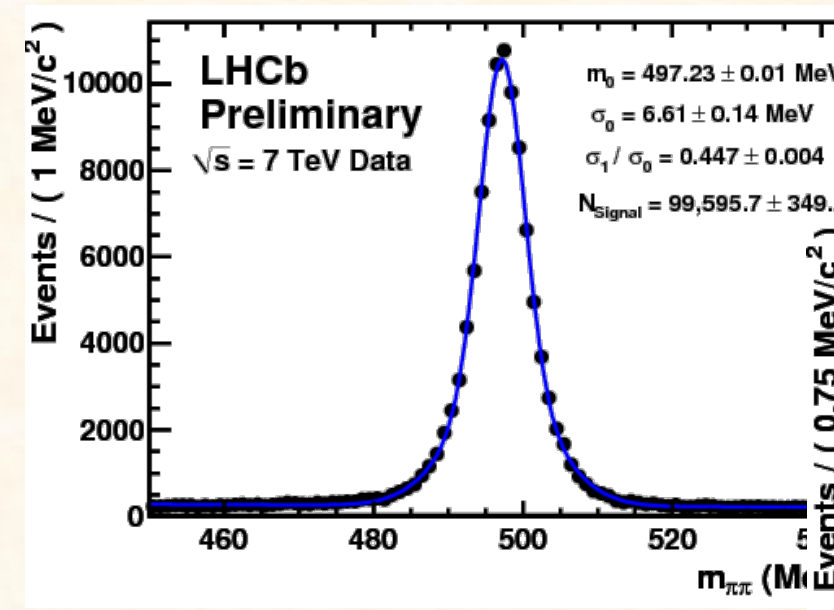

$$
\sigma=2.7 \mathrm{MeV}
$$




\section{Calorimetry performance}

\section{- Calorimetry}

$>\pi^{0} \rightarrow \mathrm{\gamma}$ mass resolution: $\sigma=7.25 \mathrm{MeV}$

$>$ clear $\mathrm{J} / \Psi \rightarrow \mathrm{e}^{+} \mathrm{e}^{-}$signal observed, $\sigma \sim 70 \mathrm{MeV}$
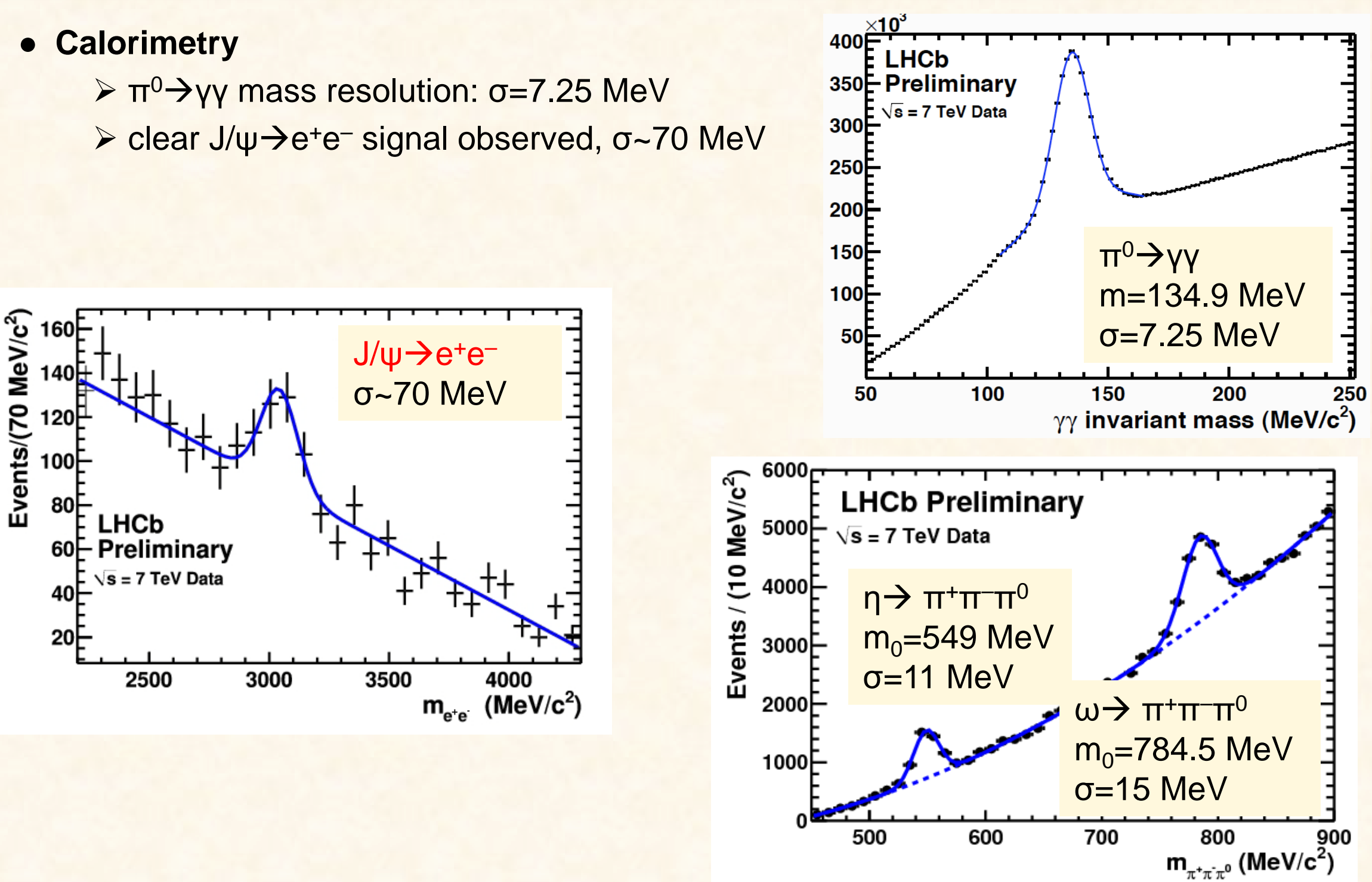


\section{Particle ID}

- Particle ID with RICH detectors

$>\mathrm{K}$ ID efficiency $\approx 96 \%, \pi \rightarrow \mathrm{K}$ misID $\approx 7 \%$
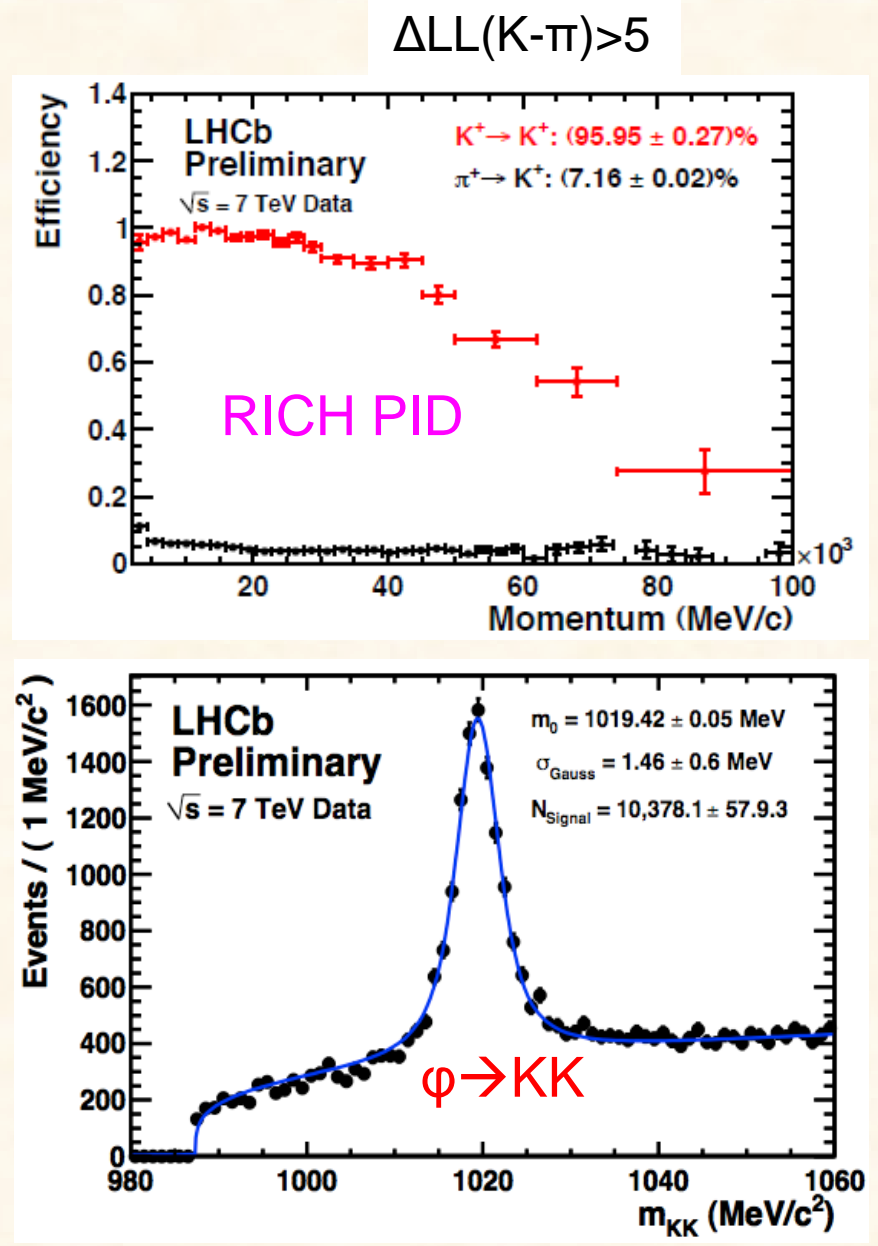

- Muon ID

$>$ efficiency $\varepsilon(\mu)=97.3 \pm 1.2 \%$

$>$ misID $\pi \rightarrow \mu \approx 2.4 \%, p \rightarrow \mu \approx 0.18 \%$
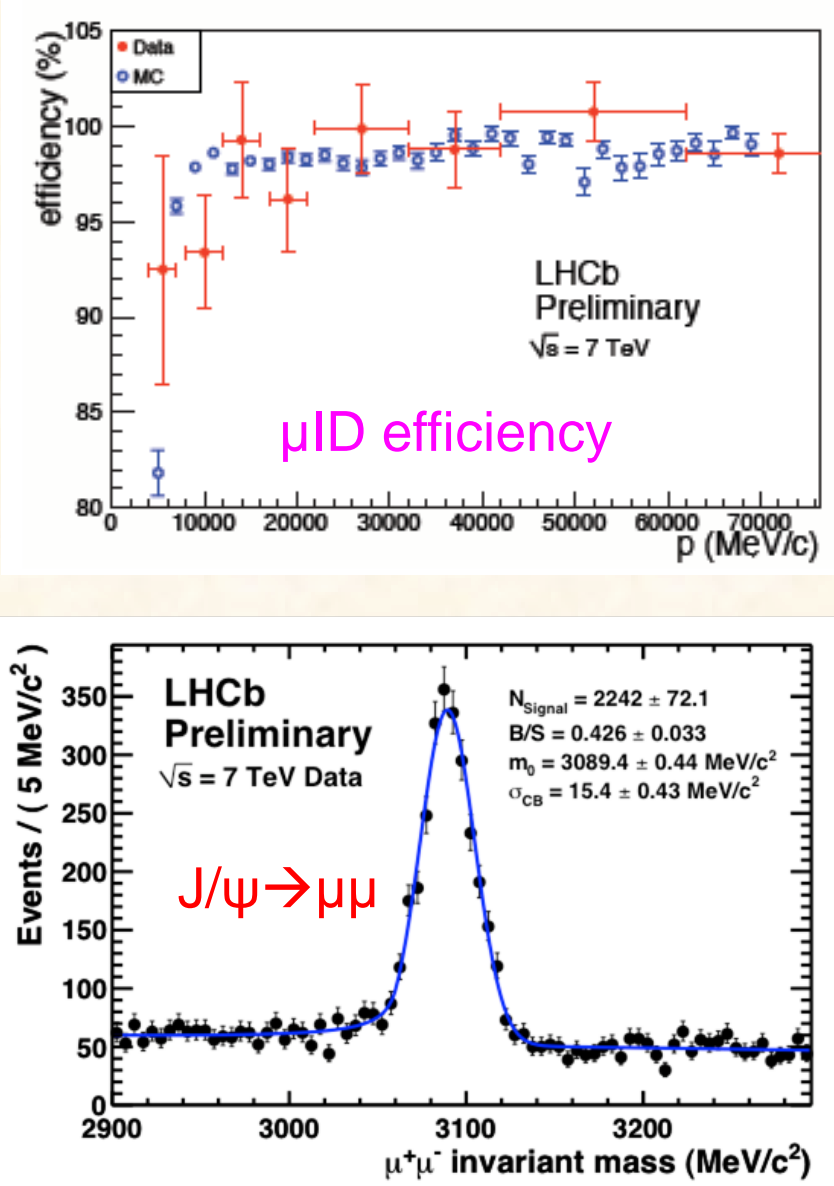


\section{$D^{0}$ lifetime}

The $\mathrm{D}$ lifetime fit gives

$\mathrm{T}(\mathrm{D} 0)=(0.398 \pm 0.026) \mathrm{ps}$,

in agreement with PDG average:

$\mathrm{T}^{\mathrm{PDG}}(\mathrm{D} 0)=(0.4101 \pm 0.0015) \mathrm{ps}$

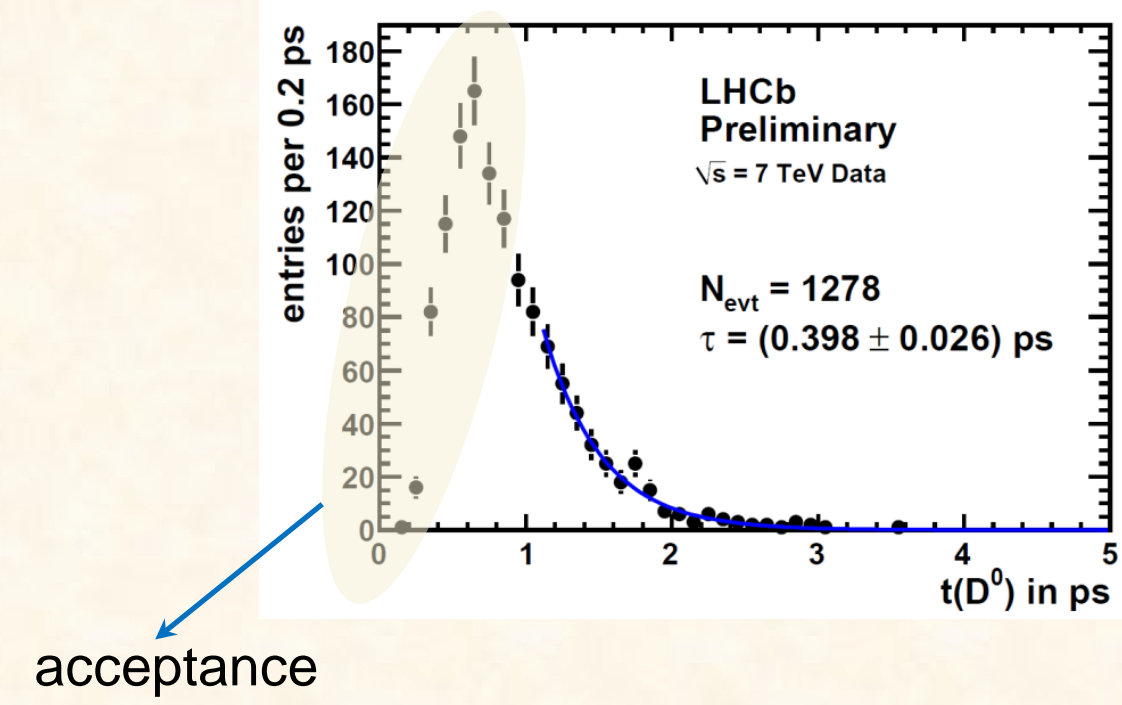




\section{LHCb run 2010-2011}

LHC is running smoothly since its startup, gradually increasing its luminosity. It is running at $\mathrm{E}_{\mathrm{CM}}=7 \mathrm{TeV}$ (the $c \bar{c}$ production cross section expected to be

\section{$\sim 0.57$ of that at $14 \mathrm{TeV}$ )}

- by now, $\mathcal{L} \approx 2 \cdot 10^{29} \mathrm{~cm}^{-2} \mathrm{~s}^{-1}$ achieved

- by the end of 2010 it is supposed to achieve:

- $\mathcal{L} \approx 10^{31} \mathrm{~cm}^{-2} \mathrm{~s}^{-1}, \int_{2010} \mathcal{L} \mathrm{dt} \approx 100 \mathrm{pb}^{-1}$

- $\sim 10^{11} c \bar{c}$ events produced

- in 2011:

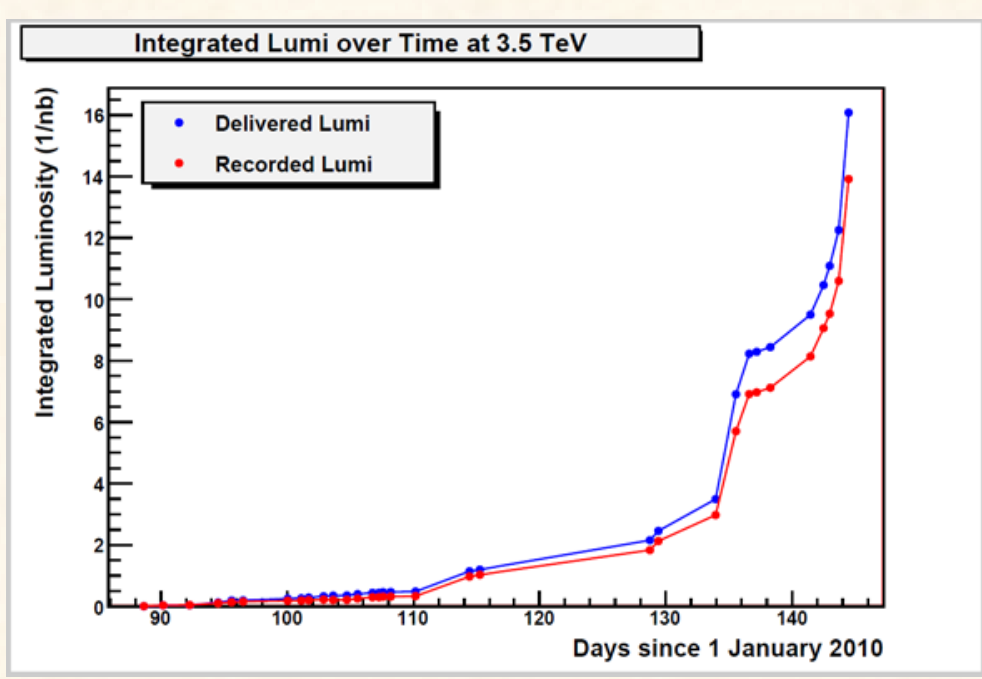

- reach $\mathrm{LHCb}$ design luminosity, $\mathcal{L} \approx 2 \cdot 10^{32} \mathrm{~cm}^{-2} \mathrm{~s}^{-1}$

- $\int_{2011} \mathcal{L} \mathrm{dt} \approx 1 \mathrm{fb}^{-1}$
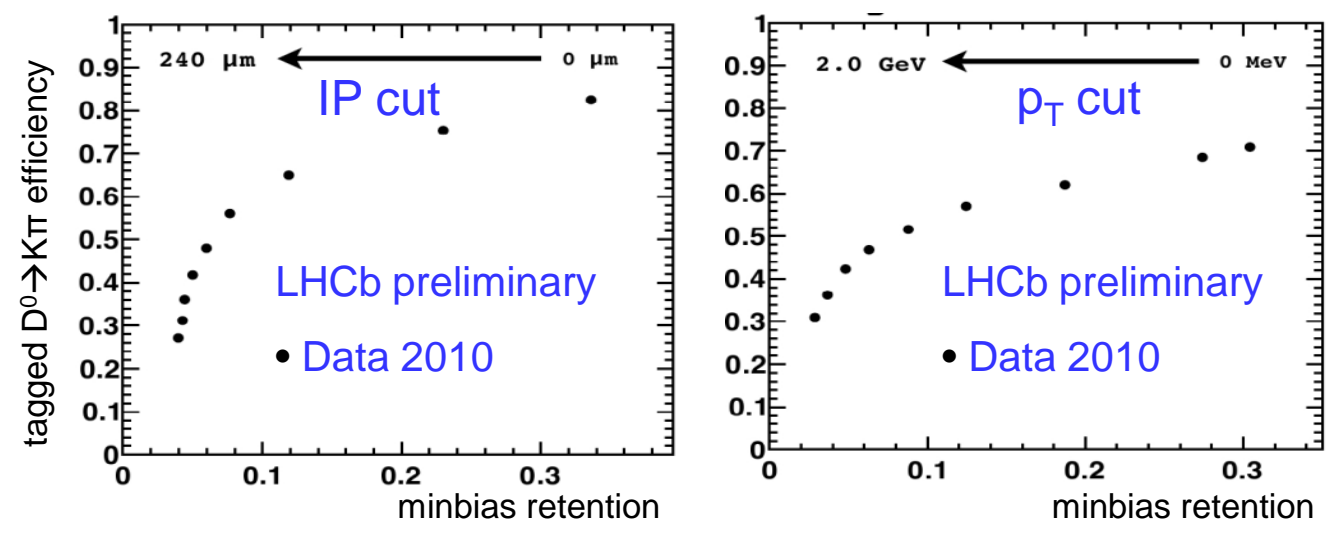

In 2010, with luminosities below few. $10^{31} \mathrm{~cm}^{-2} \mathrm{~s}^{-1}$, LHCb works with much lower trigger thresholds and factor 4-5 higher efficiencies for prompt D's $\rightarrow$ great opportunities for charm physics! 